\section{Real-world treatment outcomes in patients with metastatic Merkel cell carcinoma treated with chemotherapy in the USA}

\author{
C Lance Cowey ${ }^{* 1}$, Lisa Mahnke ${ }^{2}$, Janet Espirito ${ }^{3}$, Christoph Helwig ${ }^{4}$, \\ Dina Oksen ${ }^{4} \&$ Murtuza Bharmal ${ }^{4}$
}

\begin{abstract}
Aim: This retrospective study of patients in the USA with metastatic Merkel cell carcinoma (mMCC) aimed to assess patient responses to second-line and later (2L+) and first-line (1L) chemotherapy. Patients \& methods: Out of 686 patients with MCC identified in The US Oncology Network, 20 and 67 patients with mMCC qualified for the $2 \mathrm{~L}+$ and $1 \mathrm{~L}$ study, respectively; the primary analysis population was restricted to immunocompetent patients. Results: In the $2 \mathrm{~L}+$ primary analysis population, objective response rate (ORR) was $28.6 \%$, median duration of response (DOR) was 1.7 months and median progression-free survival was 2.2 months. In the $1 \mathrm{~L}$ primary analysis population, ORR was $29.4 \%$, median DOR was 6.7 months and median progression-free survival was 4.6 months. Conclusion: The low ORR and brief DOR underscore the need for novel therapies.
\end{abstract}

First draft submitted: 19 April 2017; Accepted for publication: 15 May 2017; Published online: 13 June 2017

Merkel cell carcinoma (MCC) is a rare, aggressive skin cancer that occurs most frequently in elderly and immunocompromised patients [1-3]. There are approximately 1500 cases of MCC per year in the USA, and the incidence has dramatically increased over the last 20 years [4]. MCC typically presents as painless growths that are clinically unremarkable in appearance and are usually found on sun-exposed areas, such as the head and neck $[2,3]$. These tumors grow rapidly and tend to metastasize early and frequently to local regions of the body, leading to a relatively poor prognosis with this aggressive disease $[2,3,5]$. Among patients diagnosed with local or regional disease, the reported rates of recurrence range from 43 to $48 \%[6,7]$. The 5 -year overall survival (OS) rate is $40 \%$ [1] and the mortality rate with MCC is greater than that with other skin cancers, including melanoma [4].

Recently, avelumab, a human IgG1 anti-programmed death-ligand 1 (PD-L1) monoclonal antibody, was approved by the US FDA as the first and only approved treatment for patients with metastatic MCC (mMCC) [8]. Before this approval, there was no evidence-based standard therapeutic regimen for mMCC. The National Comprehensive Cancer Network treatment guidelines for mMCC [9] are based on those used for small cell lung cancer, as both are aggressive and poorly differentiated cancers [10]. Treatments typically include platinum agents, such as carboplatin or cisplatin with or without etoposide or topotecan, and are associated with high toxicity [9,11]. Although MCC is generally considered a chemosensitive tumor, responses to chemotherapy in metastatic disease are

'Baylor Charles A. Sammons Cancer Center at Dallas, Texas Oncology, Dallas, TX 75246, USA

¿EMD Serono, Inc, Billerica, MA 01821, USA

${ }^{3}$ McKesson Specialty Health, The Woodlands, TX 77380, USA

${ }^{4}$ Merck KGaA, Darmstadt, Germany

*Author for correspondence: C.Cowey@usoncology.com

\section{KEYWORDS}

- chemotherapy

- immunotherapy

- Merkel cell carcinoma 
often not durable: response rates in the secondline (2L) setting range from $23 \%$ in patients with known distant metastasis to $45 \%$ in patients with unclear (nodal and/or distant) sites of metastasis $[12,13]$. In the first-line $(1 \mathrm{~L})$ or mostly $1 \mathrm{~L}$ setting, response rates range from 53 to $61 \%$ [12-17]. Regardless of the line of therapy, disease reoccurs in most patients by 6 months $[12,14,17]$. The available data are insufficient to assess the effect of chemotherapy on OS.

In approximately $80 \%$ of cases, MCC is associated with Merkel cell polyomavirus (MCPyV) infection, although MCC can also be linked to UV-induced DNA damage $[18,19]$. In healthy individuals, infection with $\mathrm{MCPyV}$ is frequent [2]; however, additional factors, including loss of immune surveillance, are required for infection to result in MCC. Consistent with the notion that MCC has an immunologic basis, MCC is over-represented in immunocompromised patients, such as individuals with HIV or certain hematologic malignancies and organ transplant recipients, who collectively comprise approximately $10 \%$ of the MCC patient population $[5,20]$. Additionally, increased levels of intratumoral $\mathrm{CD}^{+} \mathrm{T}$ cells are associated with improved survival [21]. Moreover, enhanced expression of PD-L1 and its receptor, PD-1, within the tumor microenvironment is correlated with increased tumor-infiltrating $\mathrm{CD}^{+}$and $\mathrm{CD}^{+}{ }^{+} \mathrm{T}$ cells specific to $\mathrm{MCPyV}$ oncoproteins [22,23]. Further evidence suggests that the immunologic basis of MCC is therapeutically actionable, as recent clinical data have shown durable responses in patients who received treatment with anti-PD-L1/PD-1 monoclonal antibodies [24-27]. Together, these observations underscore the importance of immune regulation in the etiology and progression of MCC as well as the potential for immune modulation in its therapeutic management.

New, alternative therapeutic approaches to $\mathrm{mMCC}$ are urgently needed, given its poor prognosis. As mentioned earlier, recently reported trials of immunotherapy in patients with MCC using anti-PD-L1/PD-1 agents are promising [24-27] and avelumab is now approved for treatment of mMCC $[8,24,25]$. In addition, the 2017 National Comprehensive Cancer Network guidelines for MCC include pembrolizumab, an anti-PD-1 agent, for treatment of disseminated disease as clinical judgment indicates [9]. However, given the rarity of the disease and poor prognosis for patients with stage IV disease, randomized head-to-head trials comparing chemotherapy with anti-PD-L1/PD-1 immunotherapy are not likely. Thus, to properly contextualize and interpret the outcomes of these single-arm clinical trials with immune checkpoint inhibitors, observational retrospective analyses are necessary. Here, we present the results of a real-world retrospective study of patients with distant mMCC in the USA who have received $2 \mathrm{~L}$ and later $(2 \mathrm{~L}+)$ or $1 \mathrm{~L}$ chemotherapy.

\section{Patients \& methods - Study objectives}

The primary objective of this study was to determine the objective response rate (ORR) achieved with $2 \mathrm{~L}+$ chemotherapy using Response Evaluation Criteria In Solid Tumors (RECIST) v1.1 as a guide [28]. Key secondary objectives included assessment of duration of response (DOR), progression-free survival (PFS), OS, time to treatment discontinuation (TTD) and durable response rate (DRR) as well as evaluation of these objectives in patients who received 1L chemotherapy. Safety was not assessed in this study. All study objectives were analyzed in the primary analysis population (immunocompetent patients only) and overall population (immunocompetent plus eligible immunocompromised patients). Institutional Review Board and Compliance/Privacy approval was obtained for the study by The US Oncology Institutional Review Board with exemption status, due to the noninterventional nature of the study.

\section{- Patient population}

Patients in this analysis were adults $\geq 18$ years of age diagnosed with distant mMCC and treated with one line (for the $1 \mathrm{~L}$ analysis) or two or more lines (for the $2 \mathrm{~L}+$ analysis) of systemic chemotherapy. The $2 \mathrm{~L}+$ cohort was derived from the qualified 1L population. Qualifying chemotherapeutic agents for distant mMCC must have included a platinum-based agent (cisplatin or carboplatin) \pm etoposide; cyclophosphamide + doxorubicin + vincristine; topotecan; gemcitabine; irinotecan; paclitaxel; nab-paclitaxel; or docetaxel. Patients with a history of any solid tumor, except basal or squamous cell carcinoma of the skin, bladder carcinoma in situ or cervical carcinoma in situ within 3 years prior to the start of treatment for MCC, were excluded from the study. In addition, patients were excluded if they were enrolled in any interventional clinical trial or previously treated with any antibody or 
drug targeting T-cell coregulatory proteins. The primary analysis population was composed solely of immunocompetent patients, although eligible patients with immunocompromised status were also considered as part of a separate analysis of the overall population. Patients were considered immunocompromised if they had a CD 4 count of $<500$ cells $/ \mathrm{mm}^{3}$ anytime in the 12 months prior to the study period or a diagnosis of HIV or select hematologic diseases (chronic lymphocytic leukemia, multiple myeloma or hypogammaglobulinemia) in the 5 years prior to study entry or during follow-up; documented organ or allogeneic stem cell transplant prior to study entry or during follow up; or select immunosuppressive treatment within 28 days prior to the date of $2 \mathrm{~L}+$ or $1 \mathrm{~L}$ chemotherapy or during follow-up.

\section{- Data collection}

Data were obtained from iKnowMed, an oncology-specific electronic health record (EHR) system maintained by McKesson Specialty Health. The system captures outpatient medical histories from community oncology practices across the USA in The US Oncology Network, which includes over 1000 physicians in practices across 19 states. Thus, these data represent multisite treatment patterns and outcomes. The Social Security Death Index was the primary source of death information, supplemented by iKnowMed data. Records from 1 November 2004 to 30 September 2014 were searched, and qualifying patients were followed up to the end of the study period (30 June 2015) unless loss to follow-up or a record of death occurred first. All data were handled in compliance with the Health Insurance Portability and Accountability Act and the Health Information Technology for Economic and Clinical Health Act.

\section{- Outcome measures \& statistical considerations}

ORR, defined as the number of patients who reached a best overall response of complete (CR) or partial response (PR) divided by the total number of patients, was based on clinical review of physician progress notes and radiology reports as available in the EHR to assess measurable disease using RECIST v1.1 as a guide. Patients without baseline measurable disease were classified as not evaluable. DOR, TTD, PFS and OS were estimated using Kaplan-Meier methodology. DRR was defined as the proportion of patients with an objective response lasting $\geq 6$ months.

\section{Results \\ - Patient population}

A total of 686 patients with MCC were identified in the iKnowMed database prior to 30 September 2014 (Figure 1). Out of 255 patients who were thought to have mMCC, only 39 had evidence of $2 \mathrm{~L}+$ chemotherapy for metastatic disease. Out of these 39 patients, 20 qualified for analysis in the $2 \mathrm{~L}+$ study (14 [70.0\%] were immunocompetent, while the remaining 6 [30.0\%] were immunocompromised).

Out of the 686 patients who were originally screened, 67 qualified for analysis in the $1 \mathrm{~L}$ study. This population included 51 (76.1\%) immunocompetent and 16 (23.9\%) immunocompromised patients.

\section{- $2 \mathrm{~L}+$ patient baseline characteristics \& treatment}

Patient baseline and disease characteristics were similar between the primary analysis population (immunocompetent) and overall patient population (immunocompetent plus immunocompromised). In the $2 \mathrm{~L}+$ primary analysis population, the median age was 75.2 years and $78.6 \%$ of patients were male (Table 1 ). Nearly all patients in the primary analysis population $(92.9 \%[\mathrm{n}=13])$ had an Eastern Cooperative Oncology Group performance status of 1 at the start of $2 \mathrm{~L}+$ therapy. Most $2 \mathrm{~L}+$ patients were initially diagnosed with stage I-III disease, and the most common primary tumor sites were the lower limb or trunk $(50.0 \%[\mathrm{n}=7])$, face $(21.4 \%[\mathrm{n}=3])$ and upper limb $(14.3 \%$ $[\mathrm{n}=2])$. At the initiation of $2 \mathrm{~L}+$ treatment, visceral metastases, defined as any site other than lymph nodes, skin or soft tissue, were present in $71.4 \%$ of primary analysis patients $(\mathrm{n}=10)$.

In the $2 \mathrm{~L}+$ primary analysis population, the median TTD among patients who received chemotherapy was 1.8 months (95\% CI: 0.3-3.3 months; range: $<0.1-5.3$ months, Table 2). All patients had discontinued treatment at the time of data collection. The most commonly cited reason for discontinuation of treatment was disease progression $(57.1 \%$ $[\mathrm{n}=8])$; for $35.7 \%$ of patients $(\mathrm{n}=5)$, toxicity was the reason for discontinuation. A variety of chemotherapy regimens were used; the most common $2 \mathrm{~L}+$ therapy was topotecan $(42.9 \%[n=6]$, Table 3$)$. The results were similar between the primary analysis and overall patient populations. 


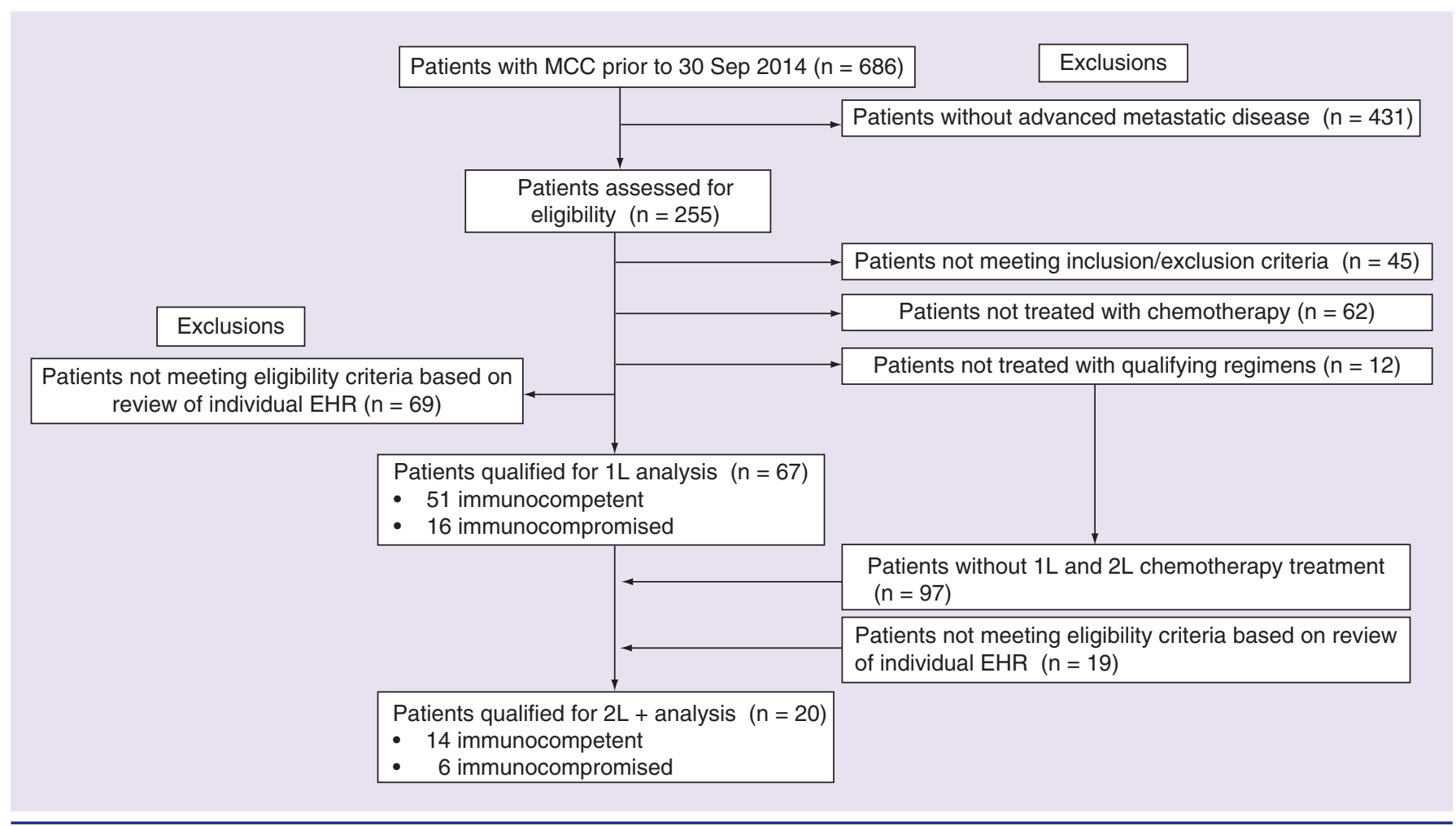

Figure 1. Patient selection.

1L: First line; 2L+: Second line and later; EHR: Electronic health record; MCC: Merkel cell carcinoma; USON: The US Oncology Network.

\section{- 1L patient baseline characteristics \&} treatment

In the $1 \mathrm{~L}$ primary analysis population, the median age was 78.1 years and $84.3 \%$ were male (Table 1). The majority $(49.0 \%[\mathrm{n}=25])$ of these patients had an Eastern Cooperative Oncology Group performance status of 1 at the start of therapy and $68.6 \%(n=35)$ were initially diagnosed with stage I-III disease. The most common primary tumor sites within this population were the lower limb or trunk $(35.3 \%[n=18])$, face $(23.5 \%[\mathrm{n}=12])$ and upper limb $(21.6 \%$ $[\mathrm{n}=11])$. At the start of $1 \mathrm{~L}$ treatment, visceral metastases were present in $66.7 \%$ of primary analysis patients $(\mathrm{n}=34)$.

The median TTD in patients who received chemotherapy was 2.4 months (95\% CI: $2.2-$ 2.9 months; range: 0.1-15.9 months; Table 2). As with patients in the $2 \mathrm{~L}+$ population, disease progression was the most common reason for discontinuation of $1 \mathrm{~L}$ treatment $(43.1 \%$ [n = 22]), although $33.3 \%$ of patients $(n=17)$ discontinued treatment due to achievement of a response. As expected, based on accepted treatment recommendations, the most commonly used treatment regimens in this population were carboplatin + etoposide $(62.7 \%[\mathrm{n}=32])$ and cisplatin + etoposide $(17.6 \%[\mathrm{n}=9])$ (Table 3$)$. The results were similar between the primary analysis and overall patient populations (Table 3).

\section{- Response to $2 \mathrm{~L}+$ chemotherapy}

No patient achieved a CR to $2 \mathrm{~L}+$ chemotherapy, although four patients (all immunocompetent) had a PR. In the primary analysis population, the ORR was $28.6 \%$ (95\% CI: $8.4-58.1 \%$ $[\mathrm{n}=4 / 14]$ ) (Table 2). Responses to chemotherapy were of limited duration in this population: the median DOR was 1.7 months (95\% CI: 0.5-3.0 months; range: 0.5-3.0 months). The median PFS was 2.2 months (95\% CI: 1.2-3.5 months) and median OS was 4.3 months (95\% CI: 2.1-6.2 months) (Figure 2). No patient had a response lasting $\geq 6$ months, and hence the DRR was $0 \%$. Results in the primary analysis population were consistent with those in the overall population (Table 2 \& Figure 2).

\section{- Response to $1 \mathrm{~L}$ chemotherapy}

In the $1 \mathrm{~L}$ overall population, 10 patients (7 immunocompetent and 3 immunocompromised) achieved a CR, while 11 patients (8 immunocompetent and 3 immunocompromised) had a PR. The ORR in the primary 


\section{Table 1. Patient and disease characteristics at baseline. ${ }^{\dagger}$}

\begin{tabular}{|c|c|c|c|c|}
\hline \multirow[t]{2}{*}{ Characteristic } & \multicolumn{2}{|c|}{$2 \mathrm{~L}+$} & \multicolumn{2}{|c|}{ 1L } \\
\hline & $\begin{array}{l}\text { Primary analysis } \\
\text { population }(n=14)\end{array}$ & $\begin{array}{l}\text { Overall population } \\
(n=20)\end{array}$ & $\begin{array}{l}\text { Primary analysis } \\
\text { population }(n=51)\end{array}$ & $\begin{array}{l}\text { Overall population } \\
(n=67)\end{array}$ \\
\hline \multicolumn{5}{|l|}{ Sex, n (\%) } \\
\hline - Male & $11(78.6)$ & $14(70.0)$ & $43(84.3)$ & $53(79.1)$ \\
\hline - Female & $3(21.4)$ & $6(30.0)$ & $8(15.7)$ & $14(20.9)$ \\
\hline \multicolumn{5}{|l|}{ Race, n (\%) } \\
\hline - White & $8(57.1)$ & $9(45.0)$ & $34(66.7)$ & $43(64.2)$ \\
\hline - Other or not documented & $6(42.9)$ & $11(55.0)$ & $17(33.3)$ & $24(35.8)$ \\
\hline \multicolumn{5}{|l|}{ Age, n (\%) } \\
\hline$-<75$ years & $7(50.0)$ & $11(55.0)$ & $21(41.2)$ & $32(47.8)$ \\
\hline$-\geq 75$ years & $7(50.0)$ & $9(45.0)$ & $30(58.8)$ & $35(52.2)$ \\
\hline Median age, years & 75.2 & 73.5 & 78.1 & 75.8 \\
\hline \multicolumn{5}{|l|}{ Stage at diagnosis, $\mathrm{n}(\%)$} \\
\hline-1 & $1(7.1)$ & $3(15.0)$ & $10(19.6)$ & $16(23.9)$ \\
\hline$-\|$ & $6(42.9)$ & $7(35.0)$ & $14(27.5)$ & $18(26.9)$ \\
\hline- III & $4(28.6)$ & $5(25.0)$ & $11(21.6)$ & $14(20.9)$ \\
\hline$-\mathrm{IV}$ & $1(7.1)$ & $1(5.0)$ & $6(11.8)$ & 7 (10.4) \\
\hline - Unknown & $2(14.3)$ & $4(20.0)$ & $10(19.6)$ & $12(17.9)$ \\
\hline \multicolumn{5}{|l|}{ Primary tumor location, $\mathrm{n}(\%)$} \\
\hline - Face & $3(21.4)$ & $5(25.0)$ & $12(23.5)$ & $16(23.9)$ \\
\hline - Lower limb or trunk & $7(50)$ & $7(35.0)$ & $18(35.3)$ & $22(32.8)$ \\
\hline - Scalp and neck & $1(7.1)$ & $2(10.0)$ & $8(15.7)$ & $12(17.9)$ \\
\hline - Unknown primary & $1(7.1)$ & $1(5.0)$ & $2(3.9)$ & $2(3.0)$ \\
\hline - Upper limb & $2(14.3)$ & $5(25.0)$ & $11(21.6)$ & $15(22.4)$ \\
\hline \multicolumn{5}{|l|}{ ECOG within 30 days prior to 10} \\
\hline days following $2 \mathrm{~L}+, \mathrm{n}(\%)$ & 0 & $1(5.0)$ & NA & NA \\
\hline-0 & $13(92.9)$ & $16(80.0)$ & NA & NA \\
\hline-1 & 0 & $1(5.0)$ & NA & NA \\
\hline-2 & $1(7.1)$ & $2(10.0)$ & NA & NA \\
\hline \multicolumn{5}{|l|}{ - Unknown } \\
\hline \multicolumn{5}{|l|}{ ECOG within 30 days prior to 10} \\
\hline days following 1L, n (\%) & NA & NA & $11(21.6)$ & $14(20.9)$ \\
\hline-0 & NA & NA & $25(49.0)$ & $32(47.8)$ \\
\hline-1 & NA & NA & $5(5.9)$ & $8(9.0)$ \\
\hline-2 or $3^{\ddagger}$ & NA & NA & 10 (19.6) & $13(19.4)$ \\
\hline - Unknown & & & & \\
\hline
\end{tabular}

analysis population was $29.4 \%(95 \% \mathrm{CI}$ : 17.5-43.8\% [n = 15/51]) (Table 2). However, durability of chemotherapy response was again modest: in the primary analysis population, the median DOR was 6.7 months $(95 \% \mathrm{CI}$ : 1.2-10.5 months; range: $0.9-63.3$ months) and DRR was $15.7 \%$ (95\% CI: $7.0-28.6 \%$ $[\mathrm{n}=8 / 51])$. The median PFS was 4.6 months (95\% CI: 2.8-7.7 months) and median OS was 10.5 months (95\% CI: 7.2-15.2 months (Figure 3). Results in the primary analysis population were consistent with those in the overall population (Table 2 \& Figure 3).

\section{Discussion}

Until the recent approval in the USA of antiPD-L1 avelumab for treatment of mMCC [8], there was no evidence-based, standard-of-care treatment for distant mMCC [9]. Because documentation of the outcomes with treatment for distant mMCC is limited [13], the aim of this real-world retrospective study was to contribute to the literature using observational historical data. Using The US Oncology Network/ McKesson Specialty Health EHR database and medical charts to assess the response to chemotherapy in patients with distant mMCC who 
Table 2. Summary of responses to chemotherapy. ${ }^{\dagger}$

\begin{tabular}{|c|c|c|c|c|}
\hline \multirow[t]{2}{*}{ Response } & \multicolumn{2}{|c|}{$2 \mathrm{~L}+$} & \multicolumn{2}{|c|}{$1 \mathrm{~L}$} \\
\hline & $\begin{array}{l}\text { Primary analysis } \\
\text { population }(n=14)\end{array}$ & $\begin{array}{l}\text { Overall population } \\
(n=20)\end{array}$ & $\begin{array}{l}\text { Primary analysis } \\
\text { population }(n=51)\end{array}$ & $\begin{array}{l}\text { Overall population } \\
(n=67)\end{array}$ \\
\hline$C R, n(\%)$ & 0 & 0 & $7(13.7)$ & $10(14.9)$ \\
\hline $\mathrm{PR}, \mathrm{n}(\%)$ & $4(28.6)$ & $4(20.0)$ & $8(15.7)$ & $11(16.4)$ \\
\hline SD, n (\%) & $2(14.3)$ & $2(10.0)$ & $1(2.0)$ & $1(1.5)$ \\
\hline PD, n (\%) & $5(35.7)$ & $8(40.0)$ & $21(41.2)$ & $31(46.3)$ \\
\hline ORR (95\% CI) (\%) & $28.6(8.4-58.1)$ & $20.0(5.7-43.7)$ & $29.4(17.5-43.8)$ & $31.3(20.6-43.8)$ \\
\hline Median DOR (95\% CI) (months) & $1.7(0.5-3.0)$ & $1.7(0.5-3.0)$ & $6.7(1.2-10.5)$ & $5.7(2.6-8.7)$ \\
\hline Range (months) & $0.5-3.0$ & $0.5-3.0$ & $0.9-63.3$ & $0.9-63.3$ \\
\hline DRR (95\% CI) (\%) & $0.0(0.0-23.2)$ & $0.0(0.0-16.8)$ & $15.7(7.0-28.6)$ & $14.9(7.4-25.7)$ \\
\hline Median TTD (95\% Cl) (months) & $1.8(0.3-3.3)$ & $1.5(0.3-2.5)$ & $2.4(2.2-2.9)$ & $2.5(2.2-3.2)$ \\
\hline
\end{tabular}

had received $2 \mathrm{~L}+$ or $1 \mathrm{~L}$ treatment, we have provided real-world clinical data representing a multisite and heterogeneous sample of patients across the USA.

In the mMCC $2 \mathrm{~L}+$ overall population, no patients achieved a CR. A PR was reported in 4 of 20 patients (20\%), all immunocompetent. The ORR for the primary analysis groups was low $(<30 \%)$. The median DOR for the overall population was very short ( $<2$ months) and median OS was poor ( $<4.5$ months).

Out of 67 patients in the $1 \mathrm{~L}$ overall population, 10 (15\%) achieved a CR (7 immunocompetent and 3 immunocompromised) and 11 (16\%) had a PR ( 8 immunocompetent and 3 immunocompromised). The objective responses achieved by immunocompromised patients suggest that, in some patients, immune system-independent mechanisms may play a role in response. However, it is difficult to assess the level of immunodeficiency in these patients based on the available data; therefore, the comparison between subgroups should be interpreted with caution. The ORR in the $1 \mathrm{~L}$ primary analysis population was low $(<30 \%)$ and median DOR was $<7$ months, similar to the ORR and median DOR in the overall population (immunocompetent plus immunocompromised). The median OS for $1 \mathrm{~L}$ ( $\leq 10.5$ months for both the primary analysis and overall population) was greater than that for $2 \mathrm{~L}+$, but remained relatively short.

In our analysis, we used RECIST as a guide; however, there are limitations to capturing traditional RECIST-based responses in the retrospective setting. In real-world clinical practice, physicians are not typically required to record treatment responses consistent with RECIST in clinical trial research. Response assessments in the real-world setting can thus be more subjective than assessments in a controlled clinical trial, and decision-making about clinical response and continuing therapy may include non-RECIST symptomatic criteria. In addition, the timing of repeat imaging studies may vary across physicians, practices and/or insurers. All evaluations in this observational study were determined by clinicians either as noted in the patient chart by the radiology scan report or the treating physician's progress notes or as interpreted by the clinician reviewer.

Despite these limitations, the results from this study are consistent with those of a recent retrospective analysis of patients with distant mMCC who received $2 \mathrm{~L}$ chemotherapy, which reported an ORR of $23 \%$ and median PFS of 2 months [12]. Similar to the results reported here, the DOR was short (median DOR of 3.3 months [range: $0.2-7.4$ months]). In addition, the results reported here are in line with a recent European observational retrospective study of 34 patients with distant mMCC enrolled in an MCC-specific registry in Germanspeaking countries, established in 2005. In this European real-world study, responses to $2 \mathrm{~L}+$ chemotherapy were of very short duration. The DOR was 1.9 months (95\% CI: $1.3-2.1$ months; range: $1.3-2.1$ months); the ORR was 8.8\% and the median PFS was 3.0 months [29].

As with the $2 \mathrm{~L}+$ findings, the results of the $1 \mathrm{~L}$ analysis are consistent with previous reports based 
Table 3. Chemotherapy regimens and treatment durations. ${ }^{\dagger}$

\begin{tabular}{|c|c|c|c|c|}
\hline \multirow[t]{2}{*}{ Regimen } & \multicolumn{2}{|c|}{ Primary analysis population $(n=14)$} & \multicolumn{2}{|c|}{ Overall population $(n=20)$} \\
\hline & $\mathbf{n}$ & $\%$ & $\mathbf{n}$ & $\%$ \\
\hline \multicolumn{5}{|l|}{$2 \mathrm{~L}+$ regimens } \\
\hline Carboplatin + etoposide & 1 & 7.1 & 1 & 5.0 \\
\hline Carboplatin + gemcitabine & 1 & 7.1 & 1 & 5.0 \\
\hline Docetaxel & 0 & 0.0 & 1 & 5.0 \\
\hline Irinotecan & 1 & 7.1 & 2 & 10.0 \\
\hline Paclitaxel & 0 & 0.0 & 1 & 5.0 \\
\hline Topotecan & 6 & 42.9 & 7 & 35.0 \\
\hline Vincristine + cyclophosphamide + doxorubicin & 4 & 28.6 & 5 & 25.0 \\
\hline Duration of $2 \mathrm{~L}+$ treatment, months & Median 1.76 & Range $0.07-5.1$ & Median 1.53 & Range $0.07-5.3$ \\
\hline Carboplatin + etoposide & 32 & 62.7 & 44 & 65.7 \\
\hline Cisplatin + etoposide & 9 & 17.6 & 11 & 16.4 \\
\hline Cisplatin + etoposide + carboplatin & 1 & 2.0 & 1 & 1.5 \\
\hline Cyclophosphamide + docetaxel & 0 & 0.0 & 1 & 1.5 \\
\hline Cyclophosphamide + doxorubicin & 1 & 2.0 & 1 & 1.5 \\
\hline Cyclophosphamide + doxorubicin + vincristine & 1 & 2.0 & 1 & 1.5 \\
\hline Gemcitabine & 1 & 2.0 & 1 & 1.5 \\
\hline Topotecan & 5 & 9.8 & 6 & 9.0 \\
\hline Duration of $1 \mathrm{~L}$ treatment, months & Median 2.4 & Range 0.1-15.9 & Median 2.53 & Range 0.1-15.9 \\
\hline
\end{tabular}

primarily on untreated patients with stage IV (distant) mMCC. The short median DOR (6.7 months) for the $1 \mathrm{~L}$ analysis in this report is in line with the median DOR found in the literature, which ranges from 3 to 10 months [12,14-17]. Additionally, the median OS (10.5 months) in the $1 \mathrm{~L}$ analysis is consistent with the median OS reported in the literature, ranging from 9 to 9.5 months in patients with distant metastatic disease $[12,15]$. The response rate in the $1 \mathrm{~L}$ population is lower than the response rate reported by Iyer et al. (31.3 vs 53\%) [12], a difference that may be attributed to how evaluable and nonevaluable patients were reported in each study. Specifically, the primary population in the current study includes results from both evaluable and nonevaluable patients, whereas the Iyer et al. study reported results from an evaluable population only [12]. In addition, the patient population in the Iyer et al. study had received no more than two lines of chemotherapy for metastatic disease [12], whereas the current study included patients who may have received more than two lines during the course of their treatment. Thus, the results of the current study add to the weight of evidence that although MCC is generally considered to be a chemosensitive tumor, the DOR is often short $[9,11-14,17,29]$ and associated with poor survival outcomes $[12,15]$. This highlights the need for new treatment options that improve the prognosis for patients with this aggressive tumor type.

MCC is considered an immunogenic cancer, as the majority of MCC cases are associated with the oncogenic MCPyV [18,19]. A relationship between MCC and immunodeficiency has been well established [5]. A strong, favorable prognostic factor in stage III MCC is 'unknown primary' status, which is believed to be linked to immune responses that resolve tumors [30]. Furthermore, a recent study found that virusnegative MCC tumors have substantial levels of neoantigens related to UV-induced DNA damage signatures, which may also result in high immunogenicity [19,31,32]. The link between survival of patients with MCC and the immune system suggests that agents that promote antitumor 
(A)

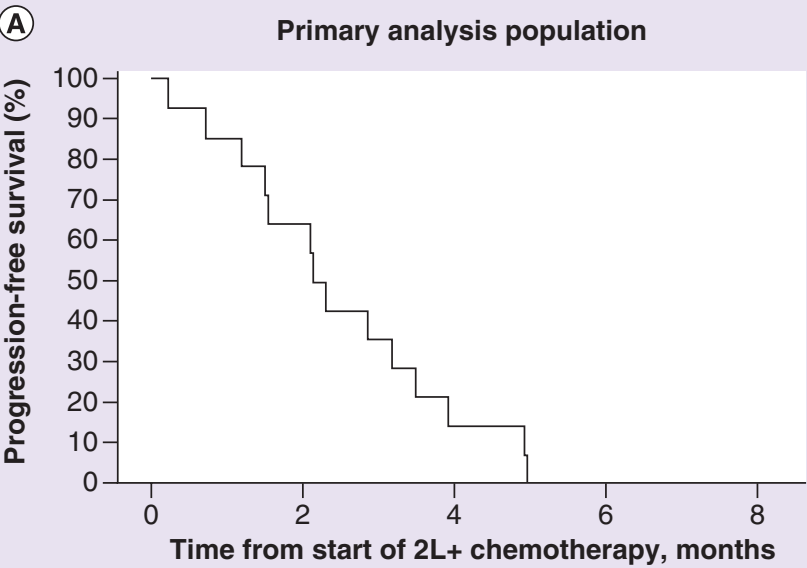

(B)

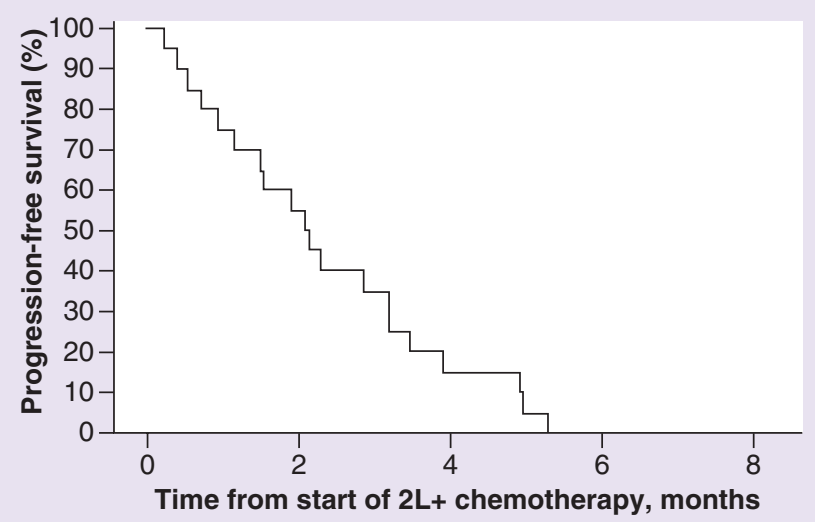

Number at risk $14 \quad 12 \quad 9 \quad 5 \quad 5 \quad 2 \quad 0$

$\begin{array}{llllll}\text { Number at risk } 20 & 15 & 11 & 7 & 3 & 1\end{array}$

(C)

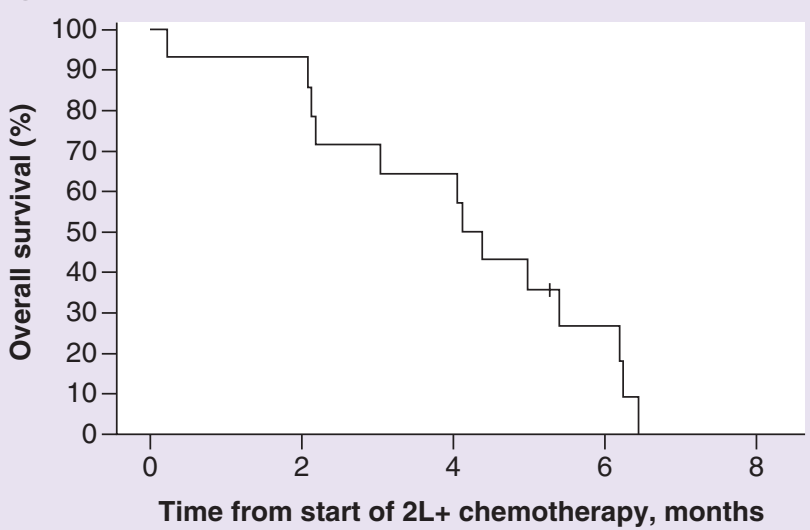

(D)

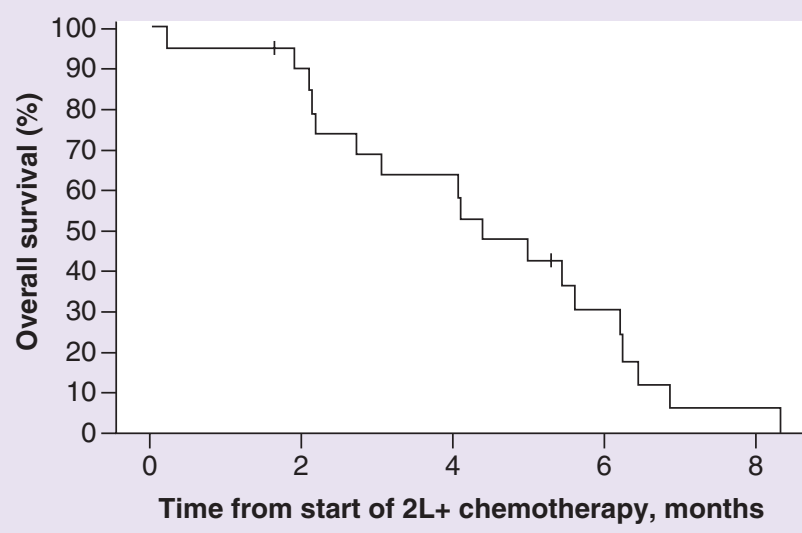

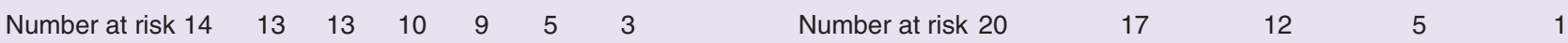

\begin{tabular}{|l|c|c|}
\hline & $\begin{array}{c}\text { Primary analysis population } \\
(\mathbf{n = 1 4 )}\end{array}$ & $\begin{array}{c}\text { Overall population }^{\dagger} \\
(\mathbf{n}=\mathbf{2 0})\end{array}$ \\
\hline Median PFS $(95 \% \mathrm{Cl})$ & $2.2(1.2-3.5)$ & $2.1(1.0-3.2)$ \\
\hline PFS rate at 6 months $(95 \% \mathrm{Cl}), \%$ & 0.0 & 0.0 \\
\hline Median OS $(95 \% \mathrm{Cl})$ & $4.3(2.1-6.2)$ & $4.4(2.2-6.2)$ \\
\hline OS rate at 6 months $(95 \% \mathrm{Cl}), \%$ & $26.8(7.3-51.5)$ & $30.2(11.6-51.4)$ \\
\hline
\end{tabular}

Figure 2. Progression-free survival and overall survival following second-line and later chemotherapy. The primary analysis population consisted of immunocompetent patients, while the overall population included both immunocompetent and immunocompromised patients.

${ }^{+}$All patients had a noted progression date; therefore, there were no censored patients.

2L+: Second line and later; Cl: Confidence interval; OS: Overall survival; PFS: Progression-free survival.

immune responses may be a beneficial treatment

option for patients with MCC [20,21,33,34]. One potential mechanism used by MCC to evade the immune system is the upregulation of immune 
(A)

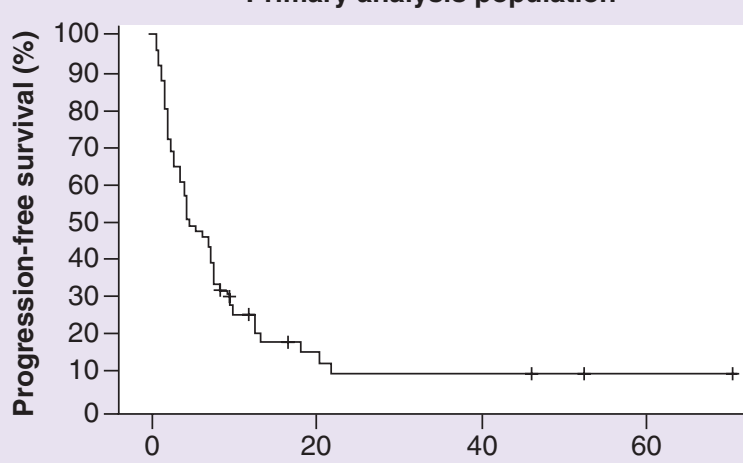

Time from start of $1 \mathrm{~L}$ chemotherapy, months
(B)

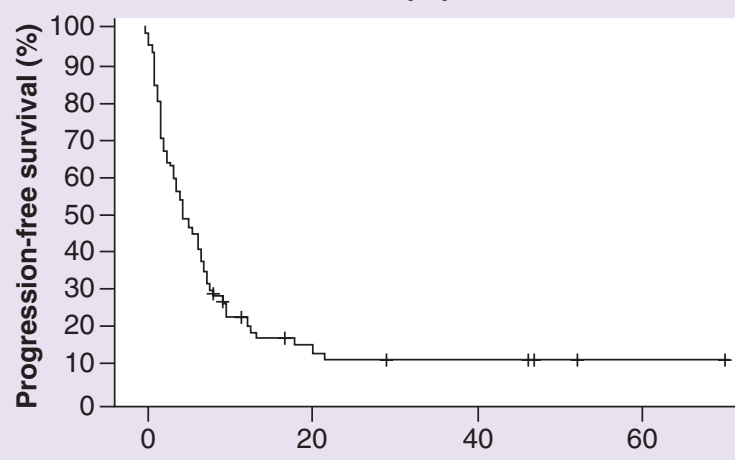

Time from start of $1 \mathrm{~L}$ chemotherapy, months

Number at risk $51 \quad 11 \quad 3 \quad \begin{array}{lllll}5 & 3 & 2 & 1\end{array}$

(C)

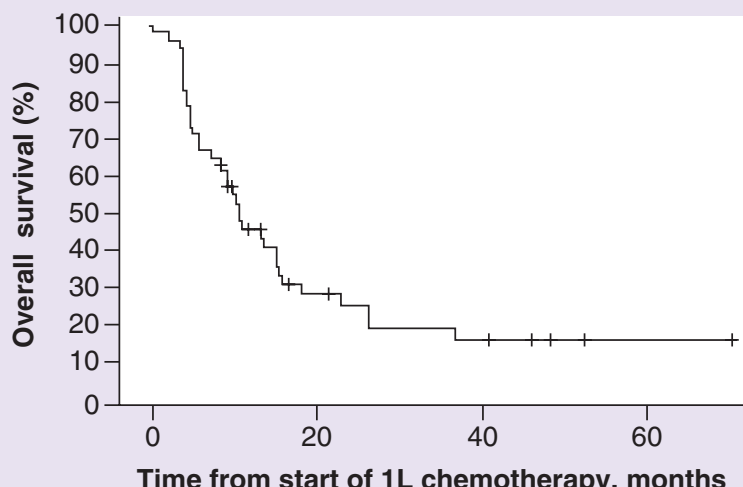

(D)

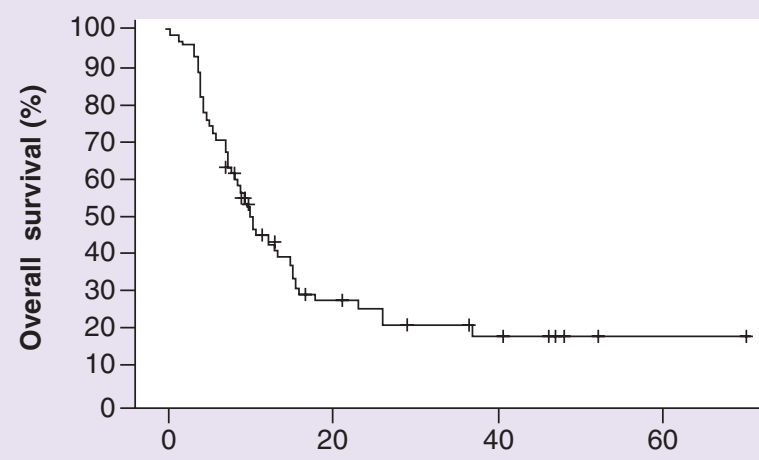

Time from start of $1 \mathrm{~L}$ chemotherapy, months

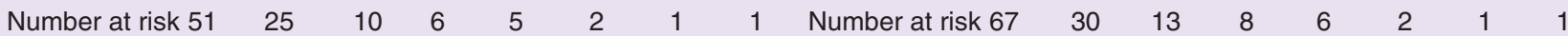

\begin{tabular}{|l|c|c|}
\hline & $\begin{array}{c}\text { Primary analysis population } \\
(\mathbf{n}=\mathbf{5 1})\end{array}$ & $\begin{array}{c}\text { Overall population } \\
(\mathbf{n}=\mathbf{6 7})\end{array}$ \\
\hline Median PFS $(95 \% \mathrm{Cl})$ & $4.6(2.8-7.7)$ & $4.6(3.0-7.0)$ \\
\hline PFS rate at 6 months $(95 \% \mathrm{Cl}), \%$ & $47.1(33.0-59.9)$ & $44.8(32.7-56.2)$ \\
\hline PFS rate at 12 months $(95 \% \mathrm{Cl}), \%$ & $24.8(13.8-37.4)$ & $21.8(12.7-32.4)$ \\
\hline PFS rate at 18 months $(95 \% \mathrm{Cl}), \%$ & $17.3(8.1-29.5)$ & $16.3(8.4-26.5)$ \\
\hline PFS rate at 24 months $(95 \% \mathrm{Cl}), \%$ & $8.7(2.4-20.0)$ & $10.2(4.0-19.7)$ \\
\hline Median OS $(95 \% \mathrm{Cl})$ & $10.5(7.2-15.2)$ & $10.2(7.4-15.2)$ \\
\hline OS rate at 6 months $(95 \% \mathrm{Cl}), \%$ & $66.7(52.0-77.8)$ & $70.1(57.5-79.5)$ \\
\hline OS rate at 12 months $(95 \% \mathrm{Cl}), \%$ & $45.3(31.0-58.6)$ & $44.0(31.5-55.8)$ \\
\hline OS rate at 18 months $(95 \% \mathrm{Cl}), \%$ & $30.2(17.5-44.0)$ & $28.7(17.7-40.7)$ \\
\hline OS rate at 24 months $(95 \% \mathrm{Cl}), \%$ & $24.4(12.7-38.3)$ & $24.5(14.1-36.4)$ \\
\hline
\end{tabular}

Figure 3. Progression-free survival and overall survival following first-line chemotherapy. The primary analysis population consisted of immunocompetent patients, while the overall population included both immunocompetent and immunocompromised patients. *Patients with data beyond the study end date were censored at the study end date of 30 June 2015.

1L: First line; OS: Overall survival; PFS: Progression-free survival. 
checkpoint regulators, such as PD-L1 [23]. It is therefore notable that inhibition of the PD-L1/ PD-1 pathway has been shown to induce durable tumor regression in $\mathrm{MCC}[24,25]$.

A Phase II trial of the anti-PD-L1 monoclonal antibody avelumab was recently conducted in patients with distant $\mathrm{mMCC}$ who had previously received 2L or later lines of treatment, leading to the approval of avelumab as a treatment for mMCC $[8,24,26]$. In this $2 \mathrm{~L}+$ study, the ORR was $32 \%$ (95\% CI: $22-43 \%)$ in the primary analysis of patients with $\geq 6$ months of follow-up [24] and $33.0 \%$ (95\% CI: $23-44 \%)$ in an updated analysis at $\geq 1$ year [26]. In contrast to the data from the current observational study of outcomes to chemotherapy, responses to avelumab were durable, with the median DOR not yet reached at the time of the updated 1-year analysis (95\% CI: 18.0 monthsnot estimable; range: 2.8-23.3+ months) [26]. Based on Kaplan-Meier analysis, the proportion of responses of $\geq 6$ months' duration was $93 \%$ (95\% CI: 74-98\%); the DRR, defined as the proportion of patients with a response lasting $\geq 6$ months and calculated as the product of the ORR and Kaplan-Meier estimate for the 6-month proportion of response duration, was 31\% (95\% CI: 21-40\%) [26]. The 6-month PFS rate was 40\% (95\% CI: 29-50\%) and the 6-month OS rate was 69\% (95\% CI: 58-78\%) [24]. At 1 year, PFS and
OS rates were $30 \%$ (95\% CI: $21-41 \%$ ) and 52\% (95\% CI: 41-62\%), respectively [26]. Enrollment of a separate cohort of chemotherapy-naive patients is currently ongoing. In another Phase II trial, the anti-PD-1 agent pembrolizumab was administered as $1 \mathrm{~L}$ treatment in patients with advanced MCC, but with limited follow-up [25]. The ORR was 56\% (95\% CI: 35-76\%) and the DOR range was from 2.2 to $\geq 9.7$ months. Based on Kaplan-Meier analysis, the 6-month PFS rate was 67\% (95\% CI: 49-86\%) [25]. Recently published data from a Phase I/II study of the antiPD-1 antibody, nivolumab, in a mixed population of chemotherapy-naive and chemotherapy-experienced patients with mMCC were consistent with the efficacy and safety findings from the avelumab and pembrolizumab studies [27]. Together, these data support anti-PD-L1/PD-1 immunotherapy as a new treatment option with the potential to meaningfully improve response duration and OS compared with existing therapies.

\section{Conclusion}

$\mathrm{mMCC}$ is a rare but aggressive disease that traditionally has lacked therapeutic options with the potential to confer durable efficacy. Our results indicate that although responses are observed with chemotherapy, duration is brief and is associated with poor OS in patients with distant mMCC.

\section{SUMMARY POINTS}

- Metastatic Merkel cell carcinoma (mMCC) is a rare and aggressive skin cancer with a poor prognosis.

- Before the recent approval of avelumab, an anti-PD-L1 monoclonal antibody, for treatment of patients with mMCC was generally limited to systemic chemotherapy.

- Observational retrospective analyses are necessary to properly contextualize and interpret the outcomes of these single-arm clinical trials with immune checkpoint inhibitors.

- This report presents the results of a real-world retrospective study of patients in the USA with distant mMCC who have received second-line $(2 \mathrm{~L})$ and later $(2 \mathrm{~L}+)$ or first-line $(1 \mathrm{~L})$ chemotherapy.

- Out of 686 identified patients with mMCC, 20 and 67 qualified for the $2 \mathrm{~L}+$ and $1 \mathrm{~L}$ study, respectively; the primary analysis population was further restricted to immunocompetent patients.

- In the $2 \mathrm{~L}+$ primary analysis population, objective response rate (ORR) was $28.6 \%$ (95\% Cl: 8.4-58.1 [n = 4/14]), median duration of response (DOR) was 1.7 months (95\% Cl: 0.5-3.0; range: 0.5-3.0), median progression-free survival was 2.2 months $(95 \% \mathrm{Cl}: 1.2-3.5)$.

- In the $1 \mathrm{~L}$ primary analysis population, ORR was $29.4 \%(95 \% \mathrm{Cl}: 17.5-43.8 \%$ [n = 15/51]), median DOR was 6.7 months (95\% Cl: 1.2-10.5; range: 0.9-63.3) and the median progression-free survival was 4.6 months (95\% Cl: 2.8-7.7).

- Results in the overall population (immunocompetent plus immunocompromised) were consistent with the primary analysis population for both $2 \mathrm{~L}+$ and $1 \mathrm{~L}$.

- The low ORR and OS and brief DOR in patients with mMCC treated with chemotherapy underscore the need for novel therapies. 
These results underscore the need for novel therapeutic approaches, with initial evidence suggesting that immune checkpoint inhibitors have the potential to dramatically improve treatment paradigms by eliciting durable responses.

\section{Acknowledgements}

The authors would like to thank the clinicians and their patients who participated in the MCC registry and the investigators in The US Oncology Network. This study was sponsored by Merck KGaA, Darmstadt, Germany, and is part of an alliance between Merck KGaA, Darmstadt, Germany and Pfizer, Inc., New York, NY, USA.

\section{Financial \& competing interests disclosure} $M$ Bharmal, C Helwig and D Oksen are employees of Merck KGaA, Darmstadt, Germany. L Mahnke is an employee of EMD Serono, Billerica, MA, USA, a business of Merck $K G a A$, Darmstadt, Germany. J Espirito is an employee of McKesson Specialty Health. Study funding was received by EMD Serono. L Cowey has received research funding from Merck KGaA, Darmstadt, Germany. C Helwig reports personal fees from Merck KGaA, Darmstadt, Germany, outside the submitted work. The authors have no other relevant affliations or financial involvement with any organization or entity with a financial interest in or financial conflict with the subject matter or materials discussed in the manuscript apart from those disclosed.

Medical writing support was provided by ClinicalThinking, Inc, Hamilton, NJ, USA and funded by Merck KGaA, Darmstadt, Germany and Pfizer, Inc., New York, NY, USA.

\section{Ethical conduct of research}

The authors state that they have obtained appropriate institutional review board approval or have followed the principles outlined in the Declaration of Helsinki for all human or animal experimental investigations. In addition, for investigations involving human subjects, informed consent has been obtained from the participants involved.

\section{Open access}

This work is licensed under the AttributionNonCommercial-NoDerivatives 4.0 Unported License. To view a copy of this license, visit http://creativecommons.org/ licenses/by-nc-nd/4.0/

\section{References}

Papers of special note have been highlighted as:

- of interest; $\bullet$ of considerable interest

1 Lemos BD, Storer BE, Iyer JG et al. Pathologic nodal evaluation improves prognostic accuracy in Merkel cell carcinoma: analysis of 5823 cases as the basis of the first consensus staging system. J. Am. Acad. Dermatol. 63(5), 751-761 (2010).

2 Saini AT, Miles BA. Merkel cell carcinoma of the head and neck: pathogenesis, current and emerging treatment options. Onco. Targets Ther. 8, 2157-2167 (2015).

3 Koljonen V. Merkel cell carcinoma. World J. Surg. Oncol. 4, 7 (2006).

4 Lemos B, Nghiem P. Merkel cell carcinoma: more deaths but still no pathway to blame. J. Invest. Dermatol. 127(9), 2100-2103 (2007).

5 Heath M, Jaimes N, Lemos B et al. Clinical characteristics of Merkel cell carcinoma at diagnosis in 195 patients: the AEIOU features. J. Am. Acad. Dermatol. 58(3), 375-381 (2008).

6 Santamaria-Barria JA, Boland GM, Yeap BY et al. Merkel cell carcinoma: 30-year experience from a single institution. Ann. Surg. Oncol. 20(4), 1365-1373 (2013).

7 Allen PJ, Bowne WB, Jaques DP, Brennan MF, Busam K, Coit DG. Merkel cell carcinoma: prognosis and treatment of patients from a single institution. J. Clin. Oncol. 23(10), 2300-2309 (2005).
8 Bavencio (avelumab) injection, package insert. Merck KGaA, Darmstadt, Germany (2017).

9 National Comprehensive Cancer Network. NCCN Clinical Practice Guidelines in Oncology: Merkel cell carcinoma. V1 (2017). www.nccn.org/professionals/physician_gls

10 Leech SN, Kolar AJ, Barrett PD, Sinclair SA, Leonard N. Merkel cell carcinoma can be distinguished from metastatic small cell carcinoma using antibodies to cytokeratin 20 and thyroid transcription factor 1. J. Clin. Pathol. 54(9), 727-729 (2001).

11 Lebbe C, Becker JC, Grob JJ et al. Diagnosis and treatment of Merkel cell carcinoma. European consensus-based interdisciplinary guideline. Eur. J. Cancer 51(16), 2396-2403 (2015).

12 Iyer JG, Blom A, Doumani R et al. Response rates and durability of chemotherapy among 62 patients with metastatic Merkel cell carcinoma. Cancer Med. 5(9), 2294-2301 (2016).

-• One of the largest observational studies on the durability of chemotherapy regimens in patients with metastatic Merkel cell carcinoma (mMCC) and the only full-length published manuscript to date to report real-world evidence data for a population of patients with confirmed metastatic MCC, including patients treated in the second-line setting.
13 Nghiem P, Kaufman H, Bharmal M, Mahnke L, Phatak H, Becker J. Systemic literature review of efficacy and safety outcomes of chemotherapy regimens in patients with metastatic MCC. Future Oncol. doi:10.2217/ fon-2017-0072 (2017) (Epub ahead of print).

-• This systemic literature review manuscript on chemotherapy outcomes in patients with mMCC is a comprehensive resource that documents a critical gap in the literature, which is the general lack of studies on outcomes associated with chemotherapy in the metastatic disease setting.

14 Tai PT, Yu E, Winquist E et al. Chemotherapy in neuroendocrine/Merkel cell carcinoma of the skin: case series and review of 204 cases. J. Clin. Oncol. 18(12), 2493-2499 (2000).

- A literature review and a case series on chemotherapy outcomes in patients with mMCC.

15 Voog E, Biron P, Martin JP, Blay JY. Chemotherapy for patients with locally advanced or metastatic Merkel cell carcinoma. Cancer 85 (12), 2589-2595 (1999).

- A literature review and a case series on chemotherapy outcomes in patients with mMCC.

16 Sharma D, Flora G, Grunberg SM. Chemotherapy of metastatic Merkel cell 
carcinoma: case report and review of the literature. Am. J. Clin. Oncol. 14(2), 166-169 (1991).

- A literature review and a case report on chemotherapy outcomes in patients with mMCC.

17 Satpute SR, Ammakkanavar NR, Einhorn LH. Role of platinum-based chemotherapy for Merkel cell tumor in adjuvant and metastatic settings. J. Clin. Oncol. 32(Suppl.), 9049 (2014)

18 Feng H, Shuda M, Chang Y, Moore PS. Clonal integration of a polyomavirus in human Merkel cell carcinoma. Science 319(5866), 1096-1100 (2008).

19 Goh G, Walradt T, Markarov V et al. Mutational landscape of MCPyV-positive and MCPyV-negative Merkel cell carcinomas with implications for immunotherapy. Oncotarget 7(3), 3403-3415 (2016).

20 Paulson KG, Iyer JG, Blom A et al. Systemic immune suppression predicts diminished Merkel cell carcinoma-specific survival independent of stage. J. Invest. Dermatol. 133(3), 642-646 (2013).

21 Paulson KG, Iyer JG, Simonson WT et al. $\mathrm{CD} 8{ }^{+}$lymphocyte intratumoral infiltration as a stage-independent predictor of Merkel cell carcinoma survival: a population-based study. Am. J. Clin. Pathol. 142(4), 452-458 (2014).

22 Iyer JG, Afanasiev OK, McClurkan C et al. Merkel cell polyomavirus-specific $\mathrm{CD} 8^{(+)}$and CD4 ${ }^{(+)} \mathrm{T}$-cell responses identified in Merkel cell carcinomas and blood. Clin. Cancer Res. 17(21), 6671-6680 (2011).

23 Afanasiev OK, Yelistratova L, Miller N et al. Merkel polyomavirus-specific $\mathrm{T}$ cells fluctuate with Merkel cell carcinoma burden and express therapeutically targetable PD-1 and Tim-3 exhaustion markers. Clin. Cancer Res. 19(19), 5351-5360 (2013).

24 Kaufman HL, Russell J, Hamid O et al. Avelumab in patients with chemotherapy- refractory metastatic Merkel cell carcinoma: a multicentre, single-group, open-label, Phase II trial. Lancet Oncol. 17(10), 1374-1385 (2016).

-. An important trial on the efficacy of avelumab, an investigational programmed death-ligand 1 inhibitor, for the treatment of patients with mMCC that provides the framework for assessing the potential of new immunotherapeutic options in the context of historical chemotherapy outcomes data, which are only available as retrospective studies.

25 Nghiem PT, Bhatia S, Lipson EJ et al. PD-1 blockade with pembrolizumab in advanced Merkel cell carcinoma. N. Engl. J. Med. 374(26), 2542-2552 (2016).

-• An important trial on the efficacy of pembrolizumab, an investigational programmed death-1 inhibitor, for the treatment of patients with advanced MCC that provides the framework for assessing the potential of new immunotherapeutic options in the context of historical chemotherapy outcomes data, which are only available as retrospective studies.

26 Kaufman HL, Russell JS, Hamid O et al. Durable responses to avelumab (anti-PD-L1) in patients with Merkel cell carcinoma progressed after chemotherapy: 1-year efficacy update. Presented at: AACR Annual Meeting 2017. Washington, DC, USA, 1-5 April 2017 (Abstract CT079).

27 Topalian SL, Bhatia S, Hollebecque A et al. Non-comparative, open-label, multiple cohort, Phase I/II study to evaluate nivolumab (NIVO) in patients with virus-associated tumors (CheckMate 358): efficacy and safety in Merkel cell carcinoma (MCC). Presented at: AACR Annual Meeting 2017. Washington, DC, USA, 1-5 April 2017 (Abstract CT074).

28 Eisenhauer EA, Therasse P, Bogaerts J et al. New response evaluation criteria in solid tumours: revised RECIST guideline (version 1.1). Eur. J. Cancer 45(2), 228-247 (2009).

29 Becker J, Lorenz E, Haas G et al. Evaluation of real world treatment outcomes in patients with metastatic Merkel cell carcinoma (MCC) following second line chemotherapy. Ann. Oncol. 26(Suppl. 3), Abstract 2602 (2016).

- An important observational study in Europe on the durability of chemotherapy regimens in patients with mMCC, including first-line and second-line and later patient populations that are highly similar to the patients qualified for analysis in the current study.

30 Harms KL, Healy MA, Nghiem P et al. Analysis of prognostic factors from 9387 Merkel cell carcinoma cases forms the basis for the new 8th edition AJCC staging system. Ann. Surg. Oncol. 23(11), 3564-3571 (2016).

31 Harms PW, Vats P, Verhaegen ME et al. The distinctive mutational spectra of polyomavirus-negative Merkel cell carcinoma. Cancer Res. 75(18), 3720-3727 (2015).

32 Wong SQ, Waldeck K, Vergara IA et al. UV-associated mutations underlie the etiology of MCV-negative Merkel cell carcinomas. Cancer Res. 75(24), 5228-5234 (2015).

33 Sihto H, Bohling T, Kavola $\mathrm{H}$ et al. Tumor infiltrating immune cells and outcome of Merkel cell carcinoma: a population-based study. Clin. Cancer Res. 18(10), 2872-2881 (2012).

34 Behr DS, Peitsch WK, Hametner C et al. Prognostic value of immune cell infiltration, tertiary lymphoid structures and PD-L1 expression in Merkel cell carcinomas. Int. J. Clin. Exp. Pathol. 7(11), 7610-7621 (2014). 